\title{
LEGUMINEUSES ARBORESCENTES POUR LA GESTION DURABLE DES TERROIRS AGRICOLES EN BASSE CÔTE D'IVOIRE
}

\author{
N'GUESSAN K. A. ${ }^{1}$ DUPUY B. $^{2}$, ASSA A. $^{3}$ et N'GORAN A. ${ }^{1}$ \\ ${ }^{1}$ Centre National de Recherche Agronomique 10 BP 1665 Abidjan 10 Côte d'Ivoire E-mail : nguessan_kanga@yahoo.fr \\ ${ }^{2}$ Centre de Coopération Internationale en Recherche Agronomique pour le Développement Campus de Baillarguet \\ 34398 Montpellier Cedex 5 France \\ ${ }^{3}$ Université de Cocody-Abidjan, Côte d'Ivoire UFR Sciences de la Terre et des Ressources Minières. Laboratoire de \\ Pédologie et Géologie Appliquée. 22 BP 582 Abidjan 22
}

\begin{abstract}
RESUME
La Basse Côte d'Ivoire connaît depuis plusieurs années, une disparition progressive des formations forestières conduisant à des jachères pauvres en ressources forestières et à des sols non propices à l'agriculture. En vue de rechercher des solutions rapides à court et moyen termes, des études ont été menées sur des légumineuses arborescentes australiennes. Des espèces ont été sélectionnées en plantation en fonction des provenances. L'Acacia mangium, Acacia crassicarpa, Acacia aulacocarpa et l'Acacia auriculiformis ont atteint des croissances importantes en 5 ans. A cet âge, les espèces ont produit une quantité suffisante de litière $\left(5 \mathrm{t} / \mathrm{ha}^{-a^{-1}}\right)$. Plantés à $3 \times 3 \mathrm{~m}$, les peuplements ont pu être traités en taillis. En association avec le cocotier et en brise-vents, les espèces ont amélioré la productivité du cocotier, protègé les cultures, délimité les propriétés foncières et produit du bois de feu et de service.
\end{abstract}

Mots-clés : Forêt, littoral, jachère, légumineuse arborescente, gestion durable, Côte d'Ivoire.

\author{
ABSTRACT \\ LEGUMINOUS TREES FOR SUSTAINABLE AGRICULTURAL SOIL MANAGEMENT \\ IN LOWER CÔTE D'IVOIRE
}

The lower Côte d'Ivoire experienced several years, a progressive disappearance of forest areas with poor fallows in forest resources and unproper soils to the agriculture. Some studies involved australian leguminous trees for short or midterm solutions. Species were been selected in plantation following their origin. Acacia mangium, Acacia crassicarpa, Acacia aulacocarpa and Acacia auriculiformis had important growth in 5 years. At this step, the species produced enough litter $\left(5 \mathrm{t} / \mathrm{ha} . \mathrm{an}^{-1}\right)$. In association with the coconut tree and as wind-screem, the species improved coconut productivity and produced fire and service wood.

Key-words : Forest, coast, fallow, leguminous tree, sustainable management, Côte d'Ivoire.

\section{INTRODUCTION}

Depuis quelques décennies, la forêt dense humide de la Côte d'Ivoire fait place à des portions de forêts en dégradation ou en reconstitution. Al'origine de cette situation, sont les activités humaines dont l'agriculture extensive et industrielle, les cultures vivrières continues, la réduction de la durée des jachères et l'exploitation forestière (Figure 1). Quant à la zone littorale, elle est caractérisée d'une part par des sables tertiaires de plus en plus dégradés pour les cultures (surtout du cocotier) et d'autre part par une pénurie intense de bois de feu et de service (Figure 2). Les problèmes consécutifs à ces situations sont multiples et exigent des solutions à court et moyen termes. Parmi elles, les plus importantes et urgentes sont : la régénération rapide et le maintien d'un 
niveau de fertilité suffisant des sols agricoles ; l'approvisionnement soutenu en bois d'œuvre, bois d'énergie et en bois de service, et la création d'états boisés réguliers et permanents pour assurer les rôles écologiques (Akomian, 1988 ; Dupuy et N'Guessan, 1990, 1991).

Les solutions envisageables doivent tenir compte d'une approche globale dans un contexte villageois et périurbain. Ainsi, depuis une vingtaine d'années, quatre légumineuses australiennes arborescentes font l'objet de sélection (provenances, espèces), d'études d'adaptabilité écologique, de croissance et de production de bois et de litière (Cabaret et N'Guessan, 1988 ; Offi, 1988 ; Dupuy et N'Guessan, 1989, 1990 ; N'Diaye, 1991 ; N'Guessan, 1991). Des associations en jachères améliorées et en mélange avec les cultures pérennes et annuelles (cocotier, palmier, maïs) ont été testées positivement en zone forestière de la Côte d'Ivoire aux niveaux des techniques culturales (Dupuy et N'Guessan, 1990, 1991), de l'amélioration de la fertilité des sols et des rendements culturaux (N'Guessan, 1990 ; Oliver et Ganry, 1994 ; Zakra, 1996 ; N'Guessan et Gnahoua, 1998 ; N'Goran, 2005) et au niveau phytopathologique (Mallet, 1988 ; Allou et al., 2001). Ces études ont fourni pour certaines, des résultats définitifs déjà applicables en milieu réel. Mais pour d'autres études, les résultats se situent au stade de diffusion en milieu réel à travers des activités de transfert d'acquis de recherche ou restent partiels avec une nécessité de recherches complémentaires. Le contenu de cet article est une contribution significative à la modélisation du comportement et de la croissance des Acacias australiens en Côte d'Ivoire

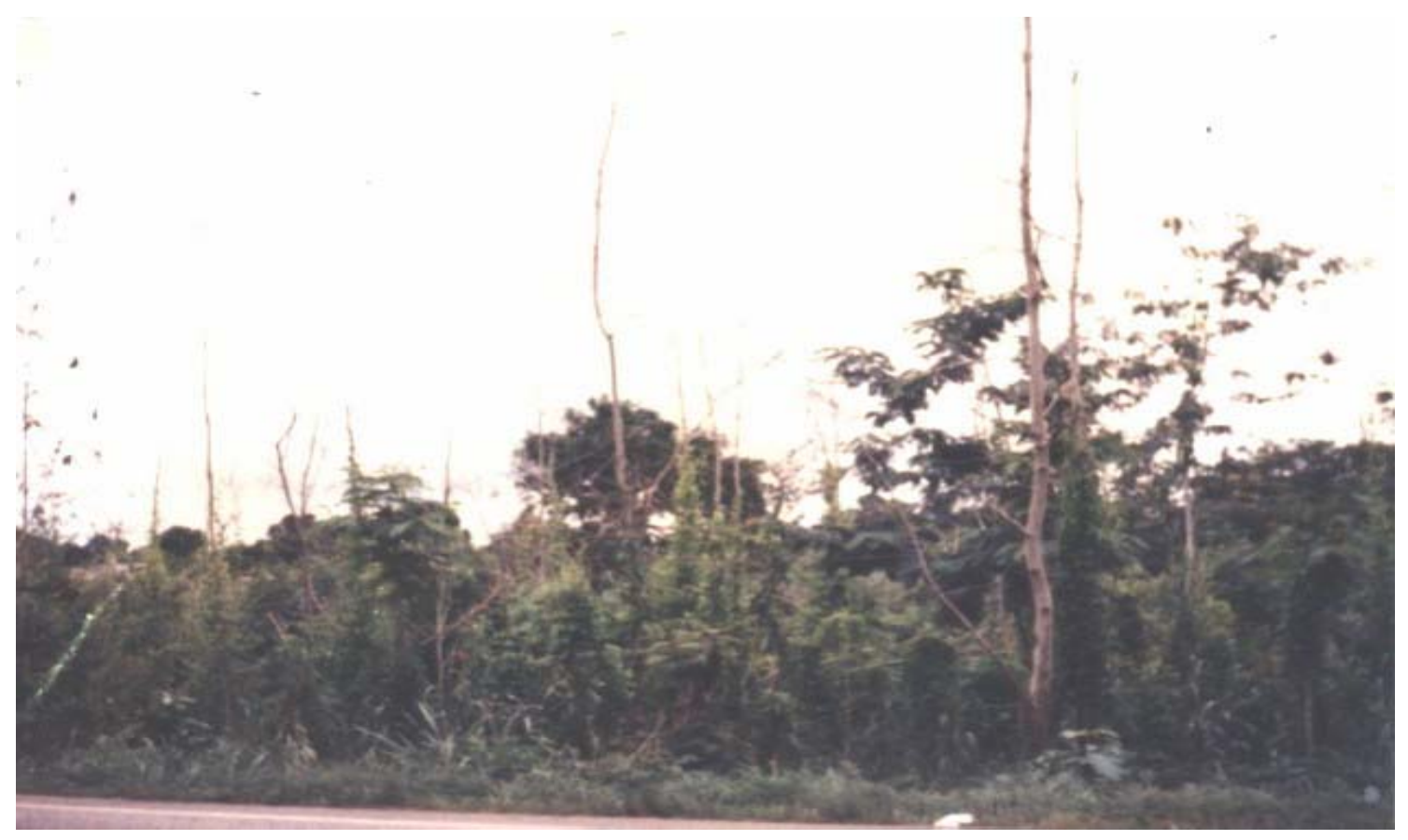

Figure 1 : Forêt dégradée suite à l'agriculture extensive.

Degradated forest as a result of extensive agriculture. 


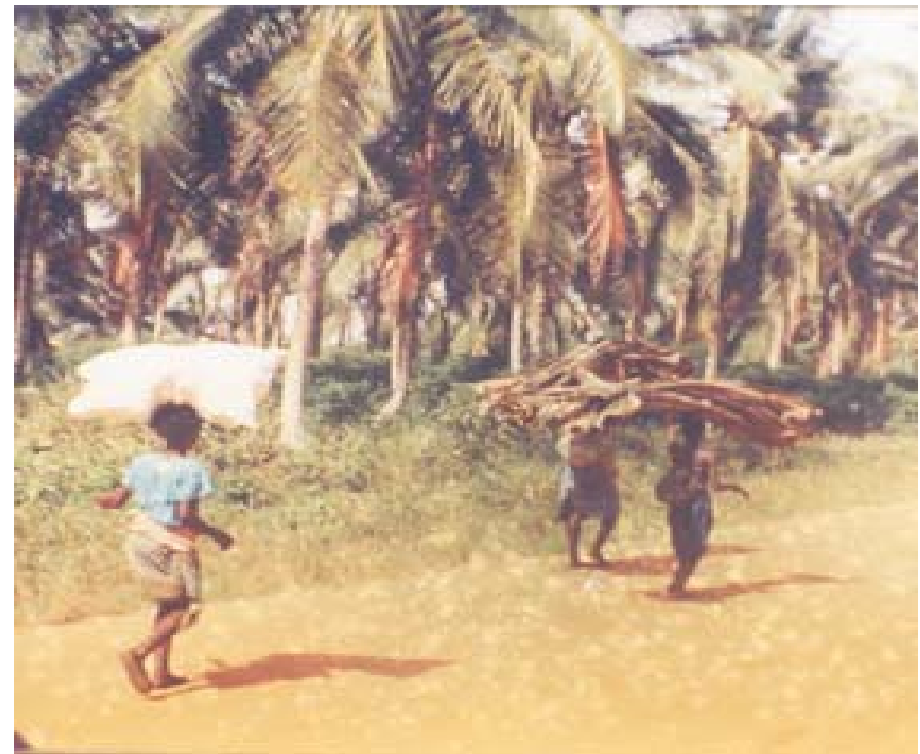

Figure 2 : Exploitation du rachis de cocotier comme bois de feu en zone littorale.

Coconut palm rachis used as fire wood in the littoral zone.

\section{MATERIEL ET METHODES}

Les études ont été réalisées en Basse Côte d'Ivoire, dans les zones de forêt dense humide sempervirente et du cordon littoral, sur six stations et sites de recherche du Centre National de Recherche Agronomique : Anguédédou, PortBouët, Assinie-France, Dabou, La Mè et Yapo. La pluviométrie moyenne annuelle est comprise entre $1525 \mathrm{~mm}$ sur le littoral (Assinie-France) et $1926 \mathrm{~mm}$ en zone de forêt dense humide sempervirente (Anguédédou). Les types de sols varient entre les sables tertiaires et quaternaires (Anguédédou, Port-Bouët, Assinie-France), les sols sablo-argileux, siliceux et ferrugineux (Anguédédou, Dabou, La Mé) et ferralitiques désaturés (Yapo).

\section{MATERIEL VEGETAL}

L'étude porte sur quatre légumineuses arborescentes: Acacia mangium, Acacia auriculiformis, Acacia crassicarpa et Acacia aulacocarpa. Elles sont originaires de l'Australie, Papouasie Nouvelle Guinée et de I'Indonésie. Les caractéristiques des arbres à l'état adulte, des aires d'origine et les usages dans ces pays de provenance sont décrits (Tableau 1) par National Academy of Science (1980), Stewart et al. (1992) et Dommergues et al. (1999). Les légumineuses arborescentes étudiées sont des espèces fixatrices d'azote atmosphérique et à croissance rapide.

Les feuilles des quatre espèces sont cotylédonaires composées et diffèrent des feuilles juvéniles et adultes du type " phyllode ». La fructification est précoce (2 à 3 ans) et le fruit est une gousse. La production de plants se fait par germination des graines prétraitées par trempage dans de l'eau bouillante pendant 30 s suivi d'un autre trempage dans l'eau froide pendant $18 \mathrm{~h}$. Les graines sont ensuite séchées à l'air libre avant le semis en sachets ou sur les planches. Le semis sur planche nécessite un repiquage ultérieur des plants. La plantation est réalisée avec des plants élevés pendant 3 à 4 mois en pépinière. 
Tableau 1 : Caractéristiques des légumineuses arborescentes et de leurs aires d'origine à l'état adulte. Characteristics of adult leguminous trees and their original areas.

\begin{tabular}{|c|c|c|c|c|c|}
\hline \multirow[b]{2}{*}{ Espèces } & \multicolumn{3}{|c|}{ Caractéristiques des aires d'origine } & \multicolumn{2}{|c|}{ Caractéristiques de l'arbre adulte } \\
\hline & $\begin{array}{l}\text { Pluviométrie } \\
(\mathrm{mm})\end{array}$ & Besoins en sol & Usages & $\begin{array}{c}\text { Diamètre } \\
\text { moyen }(\mathrm{cm})\end{array}$ & $\begin{array}{l}\text { Hauteur totale } \\
\text { moyenne }(\mathrm{m})\end{array}$ \\
\hline Acacia mangium & 1000 à 2250 & Variable & $\begin{array}{l}\text { Bois de feu } \\
\text { Construction } \\
\text { Meubles }\end{array}$ & 50 & 25 à 30 \\
\hline Acacia auriculiformis & 3000 & Variable & $\begin{array}{c}\text { Bois de feu } \\
\text { Charbon } \\
\text { Meubles } \\
\text { Tanin }\end{array}$ & 60 & 30 \\
\hline Acacia crassicarpa & 1100 & Variable & $\begin{array}{l}\text { Bois de feu } \\
\text { Construction } \\
\text { Meubles }\end{array}$ & 50 & 30 \\
\hline Acacia aulacocarpa & 500 à 4000 & Variable & $\begin{array}{l}\text { Bois de feu } \\
\text { Construction } \\
\text { Meubles }\end{array}$ & 100 & 40 \\
\hline
\end{tabular}

Sources : National Academy of Science (1980), Stewart et al. (1992), Dommergues et al. (1999).

\section{METHOES}

Plusieurs études ont été entreprises sur des thématiques différentes : sélection du matériel végétal, sylviculture, production de litière, traitement en taillis et associations culturales.

\section{Sélection du matériel végétal}

Les études réalisées visent la sélection des provenances et des espèces de légumineuses arborescentes aptes à l'introduction dans les reboisements sur la base des taux de survie, de l'état sanitaire et de la croissance.

Les études ont été effectuées dans les plantations installées en zones de forêt dense humide sempervirente et du littoral. Les superficies plantées ont été généralement inférieurs ou égales à 0,50 ha. Seize provenances ont été testées pour l'Acacia mangium, 4 pour l'Acacia auriculiformis, 4 pour l'Acacia crassicarpa et 1 provenance pour l'Acacia aulacocarpa. Les plantations ont été installées à des écartements de $3 \mathrm{~m} \times 3 \mathrm{~m}$ et $2,5 \mathrm{~m} \times 2,5 \mathrm{~m}$, soit à des densités respectives variant entre 1111 et 1600 tiges. ha $^{-1}$ (Dupuy et N'Guessan, 1989). Les paramètres privilégiés dans ces études sont le taux de survivants obtenus par comptage des plants et la croissance en diamètre à $1,30 \mathrm{~m}$ du sol et en hauteur exprimée par la circonférence à 1,30 m et la hauteur totale. Pour les plantations sous couvert de cocotiers, une dizaine de légumineuses arborescentes ont été testées sur les critères de la croissance, de la résistance aux embruns de mer et à l'ombrage. Ces espèces arborescentes appartiennent aux genres Acacia, Albizia, Leucaena et Cassia. Les paramètres retenus ont été le taux de survie, l'état sanitaire et la croissance des espèces.

\section{Etudes sylvicoles des légumineuses arborescentes en peuplement}

Les études sylvicoles ont eu pour objectif d'identifier des techniques de gestion durable et productive des légumineuses arborescentes en reboisements (site, écartement) à partir de l'évaluation et du suivi de la croissance des arbres et de la productivité de bois des peuplements forestiers.

Cette thématique regroupe trois études: le comportement en peuplement, la fertilisation en plantation et l'état sanitaire des reboisements.

L'étude de comportement des légumineuses arborescentes en plantation a été réalisée à partir d'un réseau multistationnel de placettes installées dans des peuplements de différents âges et soumis à plusieurs traitements sylvicoles. Une collecte des données a été 
réalisée dans des placettes temporaires âgées de 2 à 8 ans avec des densités variant de 1111 à 1600 tiges.ha-1, réparties sur toute la zone d'étude. Pour les études spécifiques d'écartements en plantation réalisées en zone forestière sur l'Acacia mangium et l'Acacia auriculiformis de bois, 3 écartements ont été retenus : 2,5 x 2,5 m (1600 tiges.ha-1), $3 \times 3 \mathrm{~m}$ (1111 tiges.ha $\left.^{-1}\right)$ et $4 \times 4 \mathrm{~m}$ (625 tiges.ha $\left.{ }^{-1}\right)$. Les deux paramètres utilisés dans ces études sont le diamètre à $1,30 \mathrm{~m}$ du sol et la hauteur totale (N'Guessan, 1991). Une étude de l'âge d'exploitabilité a été effectuée graphiquement à partir des données sur l'Acacia mangium et l'Acacia auriculiformis afin de déterminer la période de production maximale de bois et l'âge d'exploitabilité technique des espèces. Les paramètres utilisés sont les accroissements moyens et courants annuels (Dupuy et N'Guessan, 1990 ; N'Diaye, 1991);

L'étude de la fertilisation à la plantation a été réalisée sur l'Acacia mangium et l'Acacia auriculiformis. Le dispositif est du type blocs complets randomisés avec un écartement à la plantation de 2,5 x 2,5 m. Deux doses d'engrais NPK (10-10-18) ont été appliquées une semaine après la plantation en couronne autour des plants : 50 et $100 \mathrm{~g}$ par plant (N'Guessan, 1991). La productivité en bois a été suivie et régulièrement évaluée ;

Pour les études de protection de reboisement, des observations phytopathologiques ont été effectuées sur différents peuplements (espèces, âge) répartis dans toute la zone d'étude. Elles ont porté sur l'identification des parasites impliqués et les types de dégâts occasionnés dans les reboisements.

\section{Production de litière des légumineuses arborescentes}

L'objectif de cette étude réalisée est d'identifier les capacités des légumineuses arborescentes à améliorer les caractéristiques organiques des sols agricoles à travers la quantification de la litière restituée au sol par les arbres et la détermination du rythme de restitution de cette litière.
L'étude a porté sur l'Acacia mangium en zone de forêt dense humide sempervirente. La collecte des données a été effectuée à partir de capteurs à fond de grillage de $40 \times 50 \mathrm{~cm}$ installés à 0,$0 ; 0,5 ; 1,0 ; 1,5$ et $2,0 \mathrm{~m}$ du tronc des arbres. La litière recueillie chaque $15 \mathrm{j}$ pendant une année est régulièrement étuvée à $70^{\circ} \mathrm{C}$ pendant $72 \mathrm{~h}$ puis pesée pour obtenir les poids secs (N'Guessan, 1991). Les paramètres retenus dans cette étude sont la quantité et la composition mensuelles et annuelles de la litière. Les productions totales mensuelles de litière ont été obtenues par le rapport de chaque production mensuelle cumulée des trois organes sur la production totale annuelle.

\section{Traitement en taillis des légumineuses arborescentes}

L'étude de traitement en taillis (recépage) vise à déterminer les capacités à rejeter des légumineuses arborescentes et les techniques de gestion des rejets produits afin d'obtenir une production soutenue de bois d'œuvre, bois d'énergie et de bois de service.

Les recépages ont été effectués en zone de forêt dense humide sempervirente dans des peuplements d'Acacia mangium et d'Acacia auriculiformis âgés de 2 ans. Les coupes des tiges des arbres sont réalisées à des périodes différentes (mars, juin, septembre, décembre) et à des hauteurs du sol différentes (rez-terre, $25 \mathrm{~cm}, 50 \mathrm{~cm}$ et $100 \mathrm{~cm}$ du sol). Les périodes de recépage correspondent aux 4 saisons de l'année du pays. Les paramètres étudiés sont le taux de survie des souches et la production de rejets.

\section{Associations des cultures avec les légumineuses arborescentes étudiées}

Les objectifs poursuivis par ces pratiques agroforestières sont multiples : régénérer les caractéristiques physico-chimiques des sols désaturés, lutter contre l'érosion des sols, assurer l'approvisionnement en bois de feu et de service, produire de la biomasse pour le paillage (étêtage), protéger les cultures contre 
les agents mtéorologiques (vents, orages, etc.) et sécuriser les propriétés foncières.

Deux types d'associations ont été étudiés : plantations en mélange et brise-vents.

Les plantations en mélange étudiées en zone littorale sont basées sur la coplantation des légumineuses arborescentes avec les cocotiers. Les travaux ont été effectués sur l'Acacia mangium, l'Acacia auriculiformis et l'Acacia crassicarpa. Les plantations ont été réalisées avec une double haies de légumineuses arborescentes à écartements $2 \times 3 \mathrm{~m}$ en intercalaire de 2 lignes de cocotiers. Des haies de Casuarina equisetifolia (Filao) et de cocotiers sont utilisés comme brise-vents contre les embruns de mer. Des manchons en bambou sont aussi utilisés pour protéger les jeunes plants. La collecte des données a été réalisée sur des peuplements âgés de 2 à 8 ans. Les paramètres retenus pour ces études sont les taux de brûlures et les croissances en diamètre et en hauteur. Comme corollaire, une étude d'étêtage des arbres a été réalisée en zone littorale dans des peuplements d'Acacia mangium et d'Acacia auriculiformis âgés de 5 ans. La coupe a été effectuée à plus de $1 \mathrm{~m}$ du sol pour contrôler le développement rapide en hauteur des arbres qui surciment les cocotiers et pailler la biomasse prélevée. Les observations ont porté sur le taux de survie des souches des légumineuses arborescentes (Dupuy et N'Guessan, 1991 ; Zakra, 1996);

Les brise-vents d'Acacias australiens ont été installées en zone de forêt dense humide sempervirente. Ils ont été constitués de 2 lignes d'arbres plantés à $2 \times 2 \mathrm{~m}$ en quinconce. Trois espèces ont été utilisées pendant les études : Acacia mangium, Acacia auriculiformis et Acacia crassicarpa. (Dupuy et N'Guessan, 1991 ; N'Guessan, 1996 ). Les observations ont porté sur la croissance des arbres, la réduction de la verse des cultures associées (maïs, manioc), l'occupation des terres, le marquage de propriété foncière et sur l'effet des arbres de bordure sur les cultures.

\section{RESULTATS}

\section{SELECTION DU MATERIEL VEGETAL}

Les caractéristiques des provenances sont résumées dans le tableau 2.
Chez l'Acacia mangium, pour des peuplements âgés de 5 ans, les provenances San Pédro, Oriomo River, Broken Pole Creck et Claudie River présentent un taux de survivants supérieur à $70 \%$ et un diamètre variant entre 14 et 16,5 $\mathrm{cm}$. Chez l'Acacia auriculiformis, pour des peuplements âgés de 8 ans, les provenances Coën Cape et lokwa ont donné un taux de survivants d'environ $95 \%$ et un diamètre variant entre 21 et $22 \mathrm{~cm}$. Pour le même âge, la provenance San Pédro récoltée localement, le taux de survivants a été de $82 \%$ avec un diamètre moyen de $20 \mathrm{~cm}$. Chez l'Acacia crassicarpa, à 3 ans, les provenances sont Wemenever et Oriomo River ont un taux de survivants de $80 \%$ et un diamètre variant entre 10 et $11,5 \mathrm{~cm}$. La provenance d'Acacia aulacocarpa a donné à 5 ans, un taux de survivants de $80 \%$ et un diamètre moyen de $16 \mathrm{~cm}$.

Pour les espèces testées à l'ombrage des cocotiers, après 2 ans de plantation, 5 espèces ont pu survivre. II s'agit d'Acacia mangium, Acacia auriculiformis, Leucaena leucocephala, Albizia lebbek et Cassia siamea. Leurs caractéristiques sylvicoles sont consignées dans le tableau 3 et la figure 3 .

\section{ETUDES SYLVICOLES DES LEGUMINEUSES ARBORESCENTES EN PEUPLEMENT}

Dans le cadre des études de comportement en plantation, les résultats obtenus sur la croissance des légumineuses arborescentes sont résumés dans le tableau 4 . Pour toutes les légumineuses arborescentes, la croissance initiale varie entre 2 et $3,4 \mathrm{~cm}$. nn $^{-1}$ sur le diamètre et entre 1,2 et $4,8 \mathrm{~m} \cdot \mathrm{an}^{-1}$ sur la hauteur. Cette croissance a atteint son maximum vers 3 ans $\left(2,1\right.$ à $\left.3,7 \mathrm{~cm} \cdot \mathrm{an}^{-1}\right)$ chez toutes les espèces sur tous les sites d'études.

A 5 ans, en zone forestière, Acacia mangium, Acacia crassicarpa et Acacia aulacocarpa atteignent un diamètre variant entre 12 et $17,2 \mathrm{~cm}$ et une hauteur totale entre 13,3 et $24 \mathrm{~m}$. Chez l'Acacia auriculiformis, le diamètre moyen a été de $12 \mathrm{~cm}$ et la hauteur totale moyenne de 13,3 m. En zone littorale, chez l'Acacia mangium, l'Acacia crassicarpa et l'Acacia aulacocarpa, un diamètre moyen de $12 \mathrm{~cm}$ et une hauteur totale moyenne de $11 \mathrm{~m}$ ont été obtenus. Chez l'Acacia auriculiformis le diamètre moyen est de $10 \mathrm{~cm}$ et la hauteur totale moyenne de 9,4 m. A 8 ans, les accroissements 
Tableau 2 : Croissance et taux de survie des différentes provenances de légumineuses arborescentes. Growth and survival rates of different leguminous tree origin.

\begin{tabular}{|c|c|c|c|c|}
\hline \multirow[b]{2}{*}{ Espèces } & \multirow[b]{2}{*}{ Provenances } & \multicolumn{3}{|c|}{ Caractéristiques des peuplements } \\
\hline & & $\begin{array}{l}\text { Age } \\
\text { (ans) }\end{array}$ & $\begin{array}{l}\text { Taux de } \\
\text { survivants } \\
\text { (p.c.) }\end{array}$ & $\begin{array}{c}\text { Diamètre } \\
\text { moyen } \\
(\mathrm{cm})\end{array}$ \\
\hline \multirow{16}{*}{ Acacia mangium } & San Pédro (Côte d'Ivoire) & 5 & 95 & 15,6 \\
\hline & Oriomo River (QLD) & 5 & 80 & 14,3 \\
\hline & Claudie River (QLD) & 5 & 75 & 16,3 \\
\hline & Broken Pôle Creck (QLD) & 5 & 73 & 14,5 \\
\hline & Ellerbek Rd Cardwell (QLD) & 5 & 62 & 15,7 \\
\hline & Walsh's Pyramid (QLD) & 5 & 82 & 12,5 \\
\hline & $7 \mathrm{~km}$ See Of Mossman (NQLD) & 5 & 66 & 14,8 \\
\hline & Piru Ceram (Indonésie) & 5 & 52 & 13,7 \\
\hline & Sidei (Indonésie) & 5 & 36 & 11,1 \\
\hline & Cowley Beack Ruad (QLD) & 4 & 56 & 12,3 \\
\hline & Trinity Inlet (QLD) & 4 & 68 & 11,3 \\
\hline & Iron Range (QLD) & 8 & 40 & 23 \\
\hline & Kennedy (QLD) & 8 & 59 & 20 \\
\hline & Sud Cardwell (QLD) & 8 & 59 & 21 \\
\hline & Kuranda (QLD) & 8 & 19 & 21 \\
\hline & Innisfait (QLD) & 8 & 48 & 21 \\
\hline & Balamuk (PNG) & 8 & 83 & 20 \\
\hline Acacia & Iokwa (PNG) & 8 & 95 & 21 \\
\hline \multirow[t]{3}{*}{ auriculiformis } & Coën Cape (QLD) & 8 & 94 & 22 \\
\hline & San Pédro (Côte d'Ivoire) & 8 & 82 & 20 \\
\hline & Wemenever (PNG) & 3 & 80 & 11,6 \\
\hline Acacia & Daintree (QLD) & 3 & 78 & 8,2 \\
\hline \multirow[t]{2}{*}{ crassicarpa } & Oriomo River (PNG) & 3 & 80 & 10,1 \\
\hline & Sud de Musgrave (QLD) & 3 & 58 & 7,9 \\
\hline $\begin{array}{l}\text { Acacia } \\
\text { aulacocarpa }\end{array}$ & & 5 & 80 & 16 \\
\hline
\end{tabular}

Tableau 3 : Croissance des légumineuses arborescentes à 2 ans sous cocotiers (Port-Bouët). Growth of 2 year-aged leguminous trees intercropped with coconut at Port-Bouët.

\begin{tabular}{lcc}
\hline Espèces & $\begin{array}{c}\text { Diamètre moyen au collet } \\
(\mathrm{cm})\end{array}$ & $\begin{array}{c}\text { Hauteur totale moyenne } \\
(\mathrm{m})\end{array}$ \\
\hline Acacia mangium & 2,3 & 1,9 \\
Acacia auriculiformis & 2,6 & 2,4 \\
Cassia siamea & 2,0 & 2,4 \\
Leucaena leucocephala & 1,1 & 1,5 \\
Albizia lebbek & 2,5 & 2,5 \\
\hline
\end{tabular}

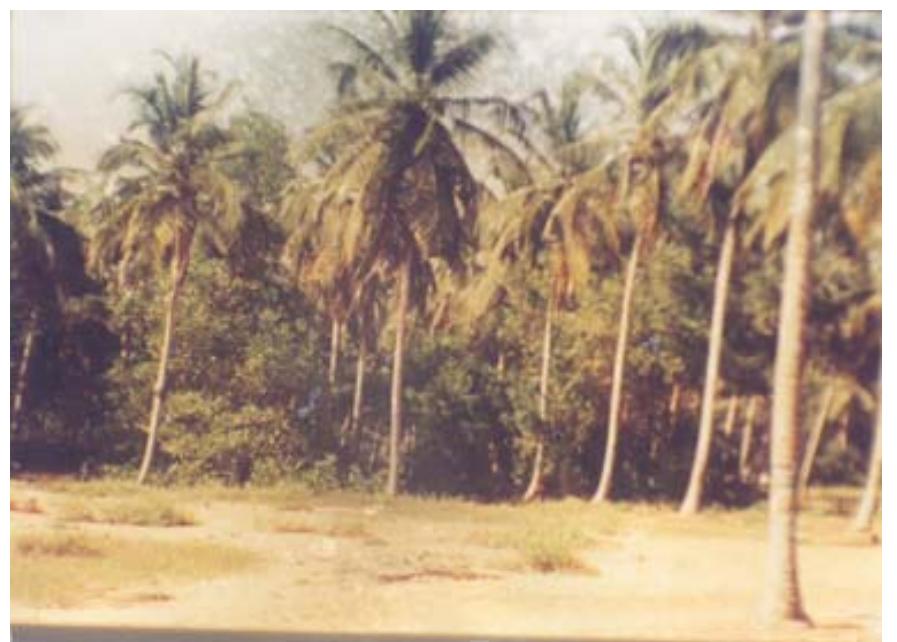

Figure 3 : Plantation de légumineuses arborescentes sous cocoteraie (Port-Bouët). Leguminous trees intercropped with coconut at Port-Bouët. 
Tableau 4 : Diamètre et hauteur des légumineuses arborescentes étudiées.

Height and diameter of studied leguminous tree species.

\begin{tabular}{|c|c|c|c|c|c|}
\hline \multirow[b]{2}{*}{ Espèces } & \multirow[b]{2}{*}{$\begin{array}{l}\text { Age } \\
\text { (ans) }\end{array}$} & \multirow{2}{*}{$\begin{array}{l}\text { Diamètre } \\
\text { moyen } \\
(\mathrm{cm})\end{array}$} & \multirow{2}{*}{$\begin{array}{l}\text { Hauteur totale } \\
\text { moyenne } \\
(\mathrm{m})\end{array}$} & \multicolumn{2}{|c|}{ Accroissements moyens annuels } \\
\hline & & & & $\begin{array}{c}\text { Diamètre } \\
\text { (cm/an) }\end{array}$ & $\begin{array}{l}\text { Hauteur totale } \\
(\mathrm{m} / \mathrm{an})\end{array}$ \\
\hline \multicolumn{6}{|c|}{ Zone forestière } \\
\hline \multirow{5}{*}{ Acacia mangium } & 2 & 6,1 & 6,3 & 3,0 & 3,1 \\
\hline & 3 & 9,5 & 11,2 & 3,2 & 3,7 \\
\hline & 5 & 15,3 & 15,8 & 3,1 & 3,2 \\
\hline & 8 & 18,4 & 21,9 & 2,3 & 2,7 \\
\hline & 2 & 5,4 & 3,7 & 2,7 & 1,8 \\
\hline \multirow{3}{*}{ Acacia auriculiformis } & 3 & 7,2 & 8,4 & 2,4 & 2,8 \\
\hline & 5 & 12,0 & 13,3 & 2,4 & 2,7 \\
\hline & 8 & 17,4 & 18,9 & 2,2 & 2,4 \\
\hline Acacia crassicarpa & 5 & 17,2 & 24,1 & 3,4 & 4,8 \\
\hline Acacia aulacocarpa & 5 & 15,6 & 19,8 & 3,1 & 4,0 \\
\hline \multicolumn{6}{|c|}{ Zone littorale } \\
\hline \multirow{4}{*}{ Acacia mangium } & 2 & 5,0 & 6,3 & 2,5 & 3,1 \\
\hline & 3 & 7,8 & 7,7 & 2,5 & 2,6 \\
\hline & 5 & 12,1 & 10,8 & 2,4 & 2,2 \\
\hline & 8 & 17,0 & 18,0 & 2,1 & 2,2 \\
\hline \multirow{4}{*}{ Acacia auriculiformis } & 2 & 4,0 & 2,4 & 2,0 & 1,2 \\
\hline & 3 & 6,3 & 6,3 & 2,1 & 2,1 \\
\hline & 5 & 10,0 & 9,4 & 2,0 & 1,9 \\
\hline & 8 & 15,6 & 15,0 & 1,9 & 1,9 \\
\hline \multirow{3}{*}{ Acacia crassicarpa } & 2 & 6,0 & 5,2 & 3,0 & 2,6 \\
\hline & 3 & 9,9 & - & 3,3 & - \\
\hline & 7 & 15,1 & 23,5 & 2,1 & 3,3 \\
\hline
\end{tabular}

sur le diamètre sont de $1,9 \mathrm{~cm} \cdot \mathrm{an}^{-1}$ chez l'Acacia auriculiformis et $2,1 \mathrm{~cm} \cdot \mathrm{an}^{-1} \mathrm{chez}$ les autres espèces.

Dès l'âge de 3 ans, des attaques de Cérambycides sous forme de forages sous corticaux à la base des troncs et aux collets des arbres sont observées. Elles se traduisent par des gommoses qui entraînent parfois des chablis et la mort de la racine pivotante. Pakystola mamillata est l'insecte le plus rencontré pendant ces observations (Mallet, 1988).

L'étude de la fertilisation à la plantation des légumineuses arborescentes avec l'engrais NPK (10-10-18) a donné les résultats consignés dans le tableau 5. Chez l'Acacia mangium, à 7 mois, on atteint une hauteur totale moyenne supérieur à $2 \mathrm{~m}$ et à 2 ans, l'accroissement en volume de bois se situe entre 43 et $48 \mathrm{~m}^{3} /$ ha. $a n^{-1}$ pour les doses respectives de 50 et 100 grammes/plant. Cela correspond à une augmentation de la croissance en volume de bois respectivement de $10 \%$ et $23 \%$ par rapport au témoin sans fumure. Chez l'Acacia auriculiformis, à 7 mois, la hauteur totale moyenne a été comprise entre 2,44 et 2,69 m et à 2 ans, l'accroissement en volume a varié entre 49 et $51 \mathrm{~m}^{3} / \mathrm{ha} \cdot$ an $^{-1}$ pour les mêmes doses. La croissance en volume de bois est de $24 \%$ avec l'apport de 100 grammes d'engrais/plant. Chez l'Acacia mangium, la croissance en hauteur a été augmentée de $10 \%$ à 4 mois et de 5 à $7 \%$ à 7 mois.

Les résultats concernant l'étude de la croissance des légumineuses arborescentes en fonction des écartements en plantation, à 3 ans, pour l'Acacia mangium, les accroissements sur le diamètre ont varié entre $3 \mathrm{~cm}$.an ${ }^{-1}$ pour les écartements serrés $(2,5 \times 2,5 \mathrm{~m}, 3 \times 3 \mathrm{~m})$ et $3,7 \mathrm{~cm}$. an ${ }^{-1}$ pour l'écartement moins serré $(4 \times 4 \mathrm{~m})$. Pour l'Acacia auriculiformis, les accroissements sur le diamètre ont varié entre $2,9 \mathrm{~cm} \cdot \mathrm{an}^{-1}$ pour les écartements serrés et $3,2 \mathrm{~cm} . \mathrm{an}^{-1}$ pour l'écartement moins serré. En général, moins de $7 \%$ de tiges multiples pour les fortes densités (1600 et 1111 tiges.ha-1 ${ }^{-1}$ ) et $43 \%$ pour la faible densité (625 tiges. ha-1) ont été obtenus. (Tableau 6)

Les résultats de l'étude de l'âge d'exploitabilité technique des espèces sont présentés dans la figure 4. II a varié entre 4 et 7 ans pour les Acacias australiens étudiés et correspond à la période de production maximale de bois des peuplements 


\section{PRODUCTION DE LITIERE DES LEGUMINEUSES ARBORESCENTES}

Les résultats des études de litière sont résumés dans le tableau 7 et les figures 5 et 6 . A 5 ans, Acacia mangium produit annuellement 5 t.ha $^{-1}$ de litière. Environ $56 \%$ de cette production de litière ont été enregistrés en grande saison sèche (décembre à mars) et $11 \%$ pendant la grande saison des pluies (mai à juillet). Les feuilles ont représenté $79 \%$ de la production annuelle de litière, les fruits $13,5 \%$ et les petites branches $7 \%$. Les chutes mensuelles de feuilles ont varié entre $69 \%$ au mois d'avril et $97 \%$ au mois de décembre. Pour les petites branches, les taux des chutes mensuelles ont été compris entre $0,4 \%$ au mois de décembre et $33 \%$ en mai. Quand aux fruits, les taux de chutes mensuelles ont varié entre 2 à $4 \%$ d'octobre à décembre et $31 \%$ en février. Les productions totales mensuelles de litière évaluées à $3 \%$ aux mois de juin et juillet, ont atteint 15 à $18 \%$ aux mois de janvier et février.

\section{TRAITEMENT EN TAILLIS DES LEGUMINEUSES ARBORESCENTES}

Les résultats des observations après 1 an de recépage sont consignés dans le tableau 8 .

Chez l'Acacia mangium, les taux de survie de souches ont varié entre 9 et $12 \%$ pour les recépages effectués de septembre à décembre et $90 \%$ pour ceux réalisés en mars. Ces taux ont été compris entre 30 et $43 \%$ pour les recépages réalisés à hauteurs 0,25 et $0,50 \mathrm{~m}$ du sol. Ceux des recépages rez-terre ont été évalués à $1 \%$. Chez l'Acacia auriculiformis, les taux de survie de souches ont varié entre 24 et $47 \%$ pour les recépages de mars à juin et sont devenus nuls pour ceux de septembre et décembre. Pour les hauteurs de coupe supérieures ou égales à $0,25 \mathrm{~m}$ du sol, ces taux ont varié entre 45 et $76 \%$ et sont devenus nuls pour les recépages au rez-terre. Pour les deux espèces, après 2 ans de recépage, le nombre moyen de rejets vivants par souche a varié entre 4 et 7 avec un diamètre moyen qui atteint $9 \mathrm{~cm}$.

Tableau 5 : Taux de survie et croissance des légumineuses arborescentes en fonction des doses d'engrais.

Survival rate and growth of leguminous trees as influenced by fertilizer rates.

\begin{tabular}{|c|c|c|c|c|c|}
\hline Espèces & Age & $\begin{array}{c}\text { Dose } \\
\text { d'engrais } \\
\text { (g/plant) }\end{array}$ & $\begin{array}{c}\text { Taux de } \\
\text { survie } \\
\text { (p.c.) }\end{array}$ & $\begin{array}{l}\text { Hauteur totale } \\
\text { moyenne } \\
\text { (m) }\end{array}$ & $\begin{array}{l}\text { Volume } \\
\text { moyen } \\
\left(\mathrm{m}^{3} / \mathrm{ha}\right)\end{array}$ \\
\hline \multirow{6}{*}{ Acacia mangium } & \multirow{3}{*}{7 mois } & 0 & 92 & 2,36 & \\
\hline & & 50 & 85 & 2,53 & \\
\hline & & 100 & 85 & 2,48 & \\
\hline & \multirow{3}{*}{2 ans } & 0 & 90 & & 39 \\
\hline & & 50 & 84 & & 43 \\
\hline & & 100 & 84 & & 48 \\
\hline \multirow{6}{*}{ Acacia auriculiformis } & \multirow{3}{*}{7 mois } & 0 & 89 & 2,44 & - \\
\hline & & 50 & 91 & 2,62 & - \\
\hline & & 100 & 92 & 2,69 & - \\
\hline & \multirow{3}{*}{2 ans } & 0 & 89 & & 41 \\
\hline & & 50 & 91 & & 49 \\
\hline & & 100 & 89 & & 51 \\
\hline
\end{tabular}


Tableau 6 : Croissance en diamètre de l'Acacia mangium et l'Acacia auriculiformis en fonction de l'écartement en plantation.

Acacia mangium and Acacia auriculiformis diameter growth with regard to tree spacing.

\begin{tabular}{|c|c|c|c|c|}
\hline Espèces & $\begin{array}{l}\text { Ecartements } \\
\text { à la plantation } \\
(\mathrm{m})\end{array}$ & $\begin{array}{l}\text { Age } \\
\text { (ans) }\end{array}$ & $\begin{array}{l}\text { Diamètre } \\
\text { moyen } \\
(\mathrm{cm})\end{array}$ & $\begin{array}{c}\text { Accroissement } \\
\text { moyen annuel sur } \\
\text { le diamètre }(\mathrm{cm} / \mathrm{an})\end{array}$ \\
\hline \multirow{10}{*}{$\begin{array}{l}\text { Acacia } \\
\text { mangium }\end{array}$} & \multirow{3}{*}{$2,5 \times 2,5$} & 2 & 5,8 & 2,9 \\
\hline & & 3 & 9,0 & 3,0 \\
\hline & & 6 & 12,6 & 2,1 \\
\hline & \multirow{3}{*}{$3 \times 3$} & 2 & 6,2 & 3,1 \\
\hline & & 3 & 9,6 & 3,2 \\
\hline & & 6 & 13,3 & 2,2 \\
\hline & \multirow{4}{*}{$4 \times 4$} & 2 & 6,4 & 3,2 \\
\hline & & 3 & 11,1 & 3,7 \\
\hline & & 6 & 16,8 & 2,8 \\
\hline & & 2 & 7,3 & 3,6 \\
\hline \multirow{8}{*}{$\begin{array}{l}\text { Acacia } \\
\text { auriculiformis }\end{array}$} & \multirow{2}{*}{$2,5 \times 2,5$} & 3 & 8,6 & 2,9 \\
\hline & & 6 & 14,7 & 2,4 \\
\hline & \multirow{3}{*}{$3 \times 3$} & 2 & 7,5 & 3,7 \\
\hline & & 3 & 9,3 & 3,1 \\
\hline & & 6 & 14,9 & 2,5 \\
\hline & \multirow{3}{*}{$4 \times 4$} & 2 & 7,2 & 3,5 \\
\hline & & 3 & 9,6 & 3,2 \\
\hline & & 6 & 16,3 & 2,7 \\
\hline
\end{tabular}

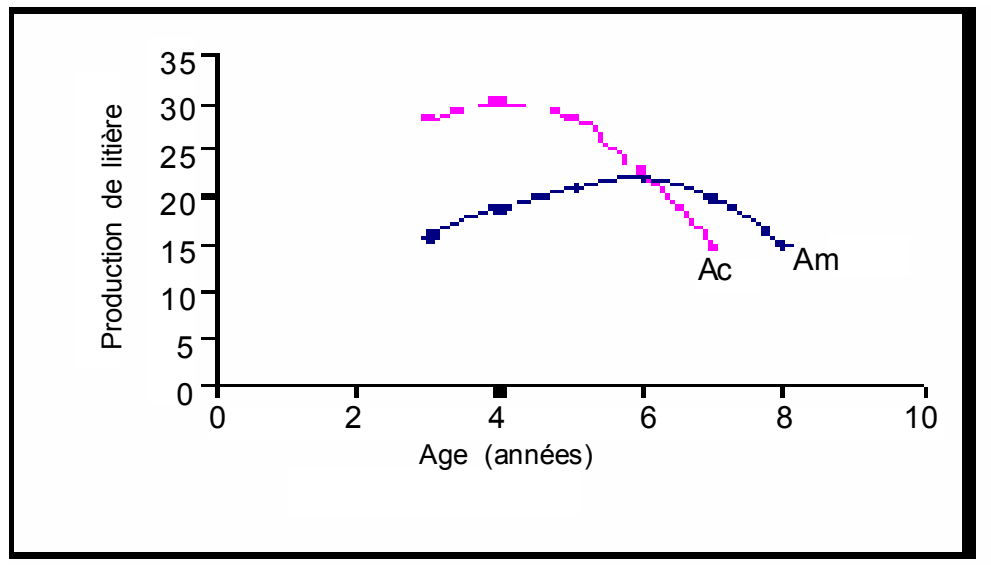

Légende : Am : Accroissement moyen

Ac : Accroissement courant

Figure 4 : Accroissements moyen et courant des légumineuses (âge d'exploitabilité).

Average and running growth of leguminous trees.

Tableau 7 : Composition et production mensuelles de litière d'Acacia mangium (\%).

Monthly production and composition of Acacia mangium litter (\%).

\begin{tabular}{lcccccccccccc}
\hline & \multicolumn{10}{c}{ Production litière (\%) } \\
\cline { 2 - 13 } Organes & janv & fév & mars & avril & mai & juin & juil & août & sept & oct & nov & déc \\
\hline Feuilles & 80 & 67 & 81 & 69 & 59 & 82 & 79 & 80 & 76 & 89 & 92 & 97 \\
Petites branches & 1 & 2 & 3 & 5 & 33 & 5 & 10 & 6 & 7 & 7 & 6 & 0,4 \\
Fruits & 19 & 31 & 16 & 26 & 8 & 13 & 11 & 14 & 17 & 4 & 2 & 3 \\
$\begin{array}{l}\text { Productions totales } \\
\text { mensuelles }\end{array}$ & 15 & 18 & 13 & 5 & 5 & 3 & 3 & 6 & 7 & 8 & 7 & 10 \\
\hline
\end{tabular}




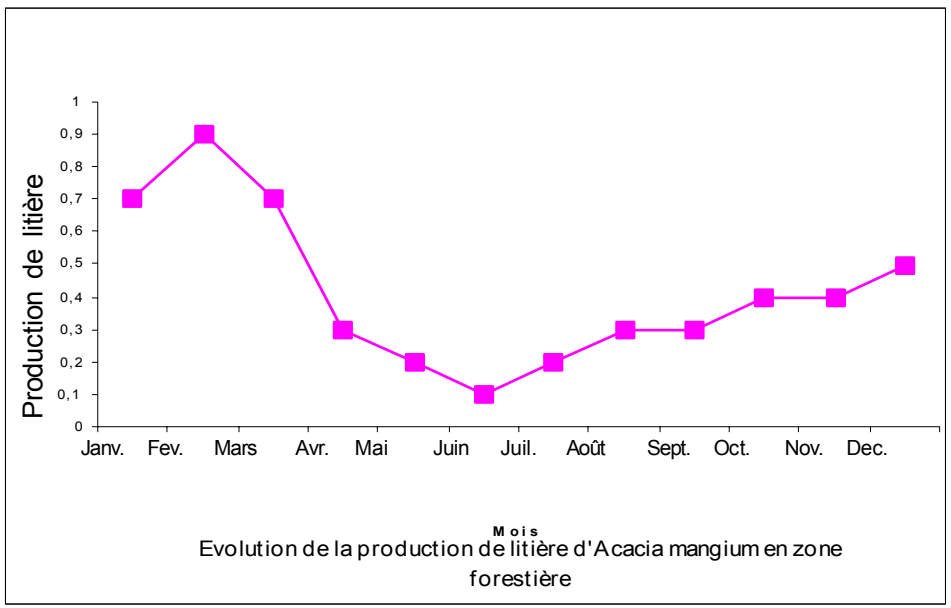

Figure 5 : Production mensuelle de litière chez Acacia mangium âgé de 5 ans (Anguédédou). Monthly litter production of five year-aged Acacia mangium at Anguédédou.

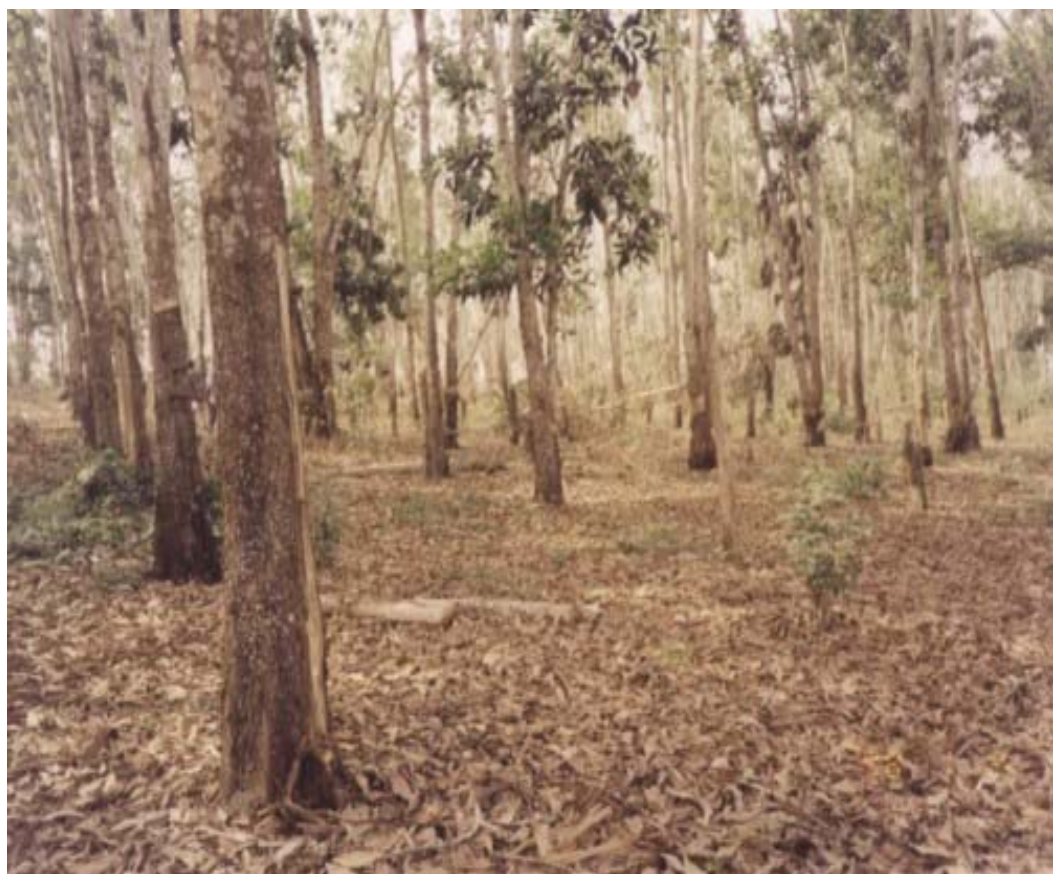

Figure 6 : Peuplement d'Acacia mangium de 17 ans avec litière en zone forestière (Dabou).

Seventeen year-aged Acacia mangium plantation with litter in the forest zone (Dabou).

Tableau 8 : Taux de survie de souches après recépage de peuplements d'Acacia mangium et d'Acacia auriculiformis âgés de 2 ans.

Stumps rates survival of Acacia mangium et Acacia auriculiformis plantings after plants cutter two years.

\begin{tabular}{lccccccccc}
\hline & Age après & \multicolumn{3}{c}{ Période de recépage } & \multicolumn{3}{c}{ Hauteur de recépage } \\
\cline { 3 - 11 } Espèces & recépage & mars & juin & sept & déc & Rez-terre & $0,25 \mathrm{~m}$ & $0,50 \mathrm{~m}$ & $1,0 \mathrm{~m}$ \\
\hline \multirow{3}{*}{ Acacia mangium } & 4 mois & 97 & 93 & 91 & 16 & 3 & 46 & 62 & \\
& 7 mois & 97 & 93 & 16 & 12 & 3 & 46 & 49 & \\
& 12 mois & 90 & 61 & 9 & 12 & 1 & 30 & 43 & 100 \\
Acacia auriculiformis & 4 mois & 73 & 38 & & & 12 & 87 & & 76 \\
& 7 mois & 59 & 20 & 27 & 18 & 5 & 50 & & 76 \\
\hline
\end{tabular}




\section{ASSOCIATIONS CULTURALES DES LEGUMINEUSES ARBORESCENTES}

\section{Association légumineuses arborescentes/cocotiers}

Les résultats de l'étude de croissance des arbres dans les associations Acacias australiens/cocotiers sont représentés dans les figures 7,8 et 9 . Pendant les deux premières années de plantation, chez l'Acacia mangium, environ 40 à $60 \%$ des plants ont présenté des brûlures aux feuilles et aux bourgeons avec la destruction totale des axes principaux. Chez l'Acacia auriculiformis, ces taux ont varié entre 20 à $30 \%$ et chez le Casuarina equisetifolia, ils sont inférieurs à $10 \%$. A 3 ans, chez l'Acacia mangium, l'accroissement sur le diamètre est de $1,8 \mathrm{~cm} \cdot \mathrm{an}^{-1}$ avec une hauteur totale de $4,5 \mathrm{~m}$ et chez l'Acacia auriculiformis, cet accroissement sur le diamètre est de $1,7 \mathrm{~cm} . \mathrm{an}^{-1}$ avec une hauteur totale de $4,8 \mathrm{~m}$. Après 4 années de plantation, la productivité de bois a été estimée à $12 \mathrm{~m}^{3} / \mathrm{ha}^{-a \mathrm{n}^{-1}}$ chez l'Acacia mangium et $4,5 \mathrm{~m}^{3} / \mathrm{ha}^{-a \mathrm{n}^{-1}}$ chez l'Acacia auriculiformis. En peuplements purs (jachères améliorées), cette productivité a été respectivement de 23 et $9 \mathrm{~m}^{3} / \mathrm{ha}^{-a n^{-1}}$ pour les 2 espèces.

L'utilisation des brise-vents de cocotiers et de Filao et des manchons de bambou baisse les effets des embruns sur une distance de 120 à $150 \mathrm{~m}$ pour l'Acacia mangium et 110 à $120 \mathrm{~m}$ pour l'Acacia auriculiformis. Sur ces distances, la croissance des arbres a été élevée et les taux de brûlures faibles. A la cinquième année de plantation des cocotiers, on note une augmentation des rendements des cocoteraies en noix de coco d'environ $50 \%$ par rapport aux cocoteraies traditionnelles et aux cocotiers hybrides sans fumure minérale (Zakra, 1996).

\section{Brise-vents des légumineuses arborescentes}

Les résultats obtenus à partir de 3 années de plantations des Acacias australiens comme brise - vents sont résumés dans la figure 10 . A 3 ans, une production de bois de maille de $12 \mathrm{~m}^{3} \cdot \mathrm{ha}^{-1}$ avec un diamètre moyen de $12 \mathrm{~cm}$ et une hauteur totale moyenne de $10 \mathrm{~m}$ chez l'Acacia mangium, l'Acacia crassicarpa et l'Acacia aulacocarpa a été obtenue. Par rapport aux cultures associées, à cet âge une mortalité des cultures de maïs et de manioc en bordure des haies sur une distance de $10 \mathrm{~m}$ pour l'Acacia mangium, l'Acacia crassicarpa et l'Acacia aulacocarpa et sur une distance de $6 \mathrm{~m}$ pour l'Acacia auriculiformis a été observée. A 8 ans, une production de bois d'environ $30 \mathrm{~m}^{3}$.ha-1 de maille chez ces espèces a été atteinte. Les productions de bois de l'Acacia auriculiformis de la maille sont inférieures à 12 et $30 \mathrm{~m}^{3}$.ha-1.

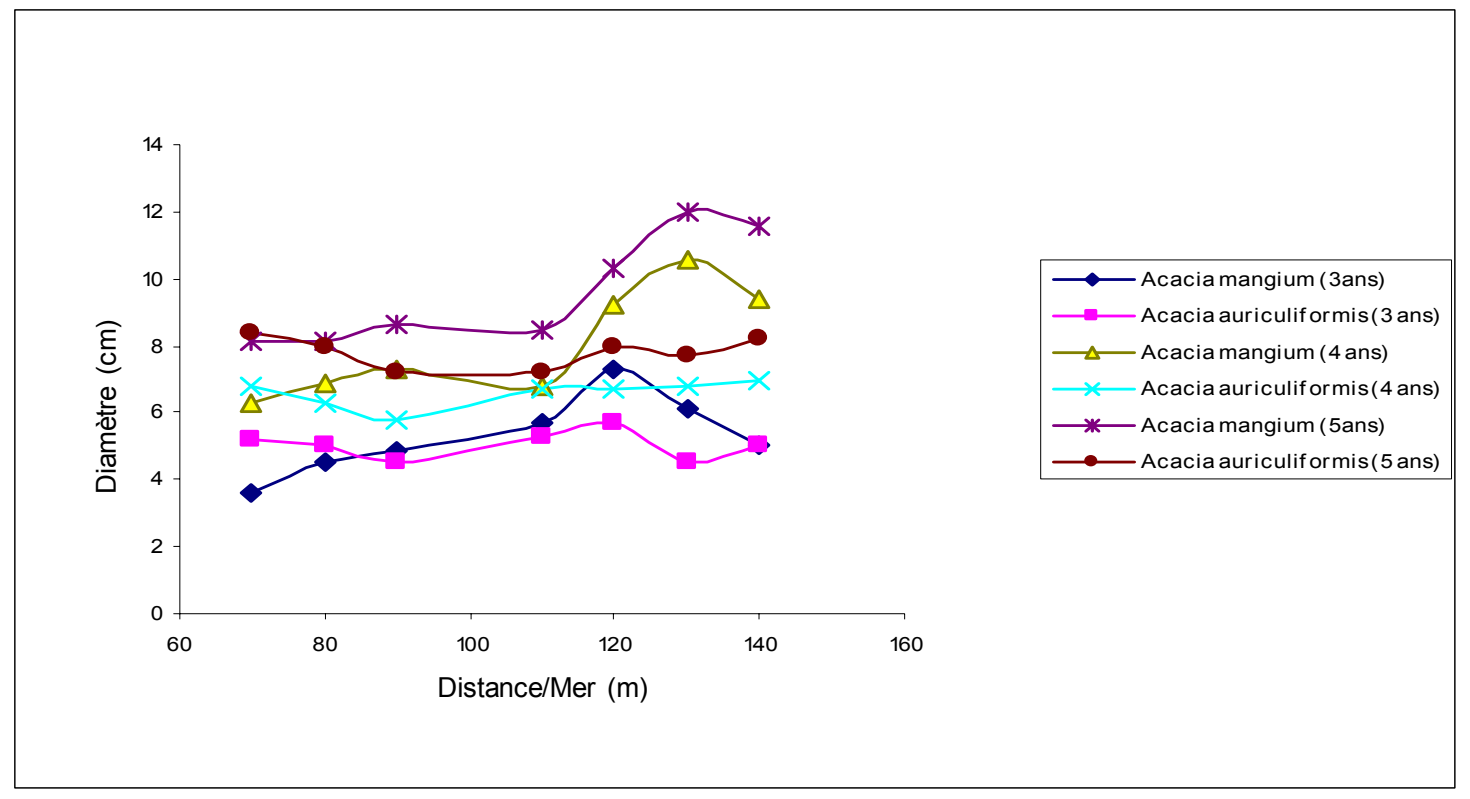

Figure 7 : Evolution du diamètre moyen des Acacias australiens en fonction de la distance à la mer.

Evolution in average diameter of Australian acacias as influenced by distance from sea. 


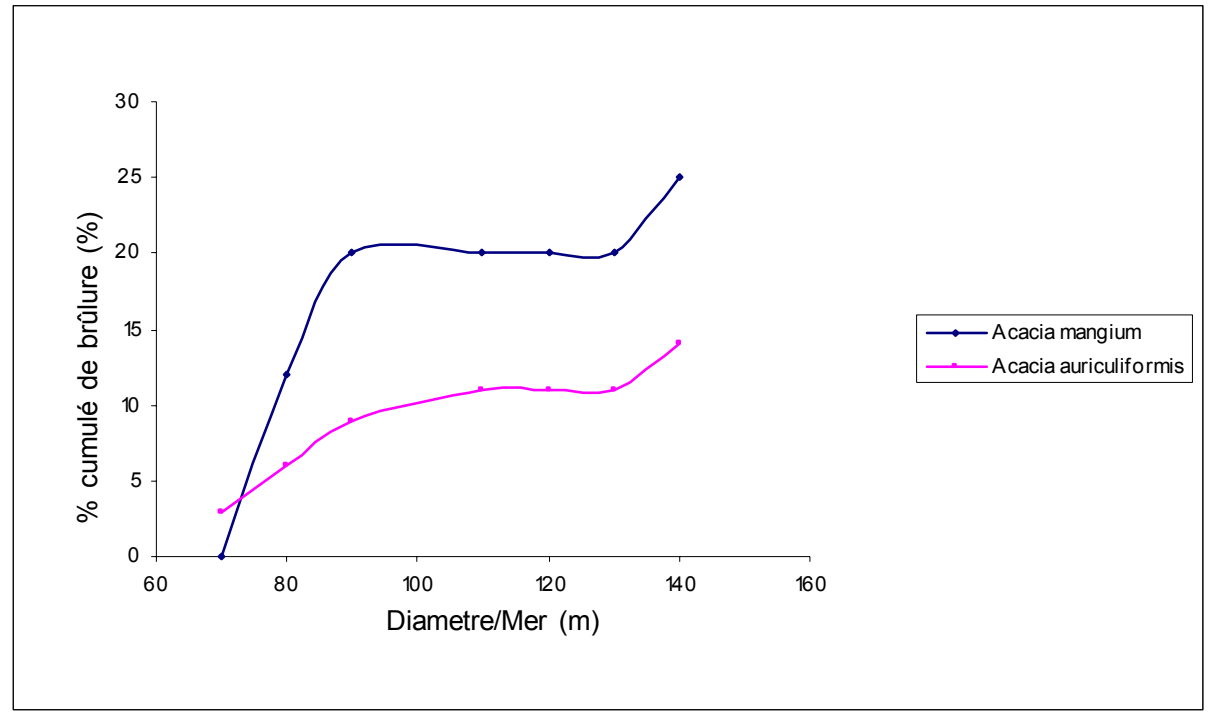

Figure 8 : Evolution du taux de brûlure des Acacias australiens en fonction de la distance à la mer. Evolution of australian Acacia burning rates with regard to distance from sea.

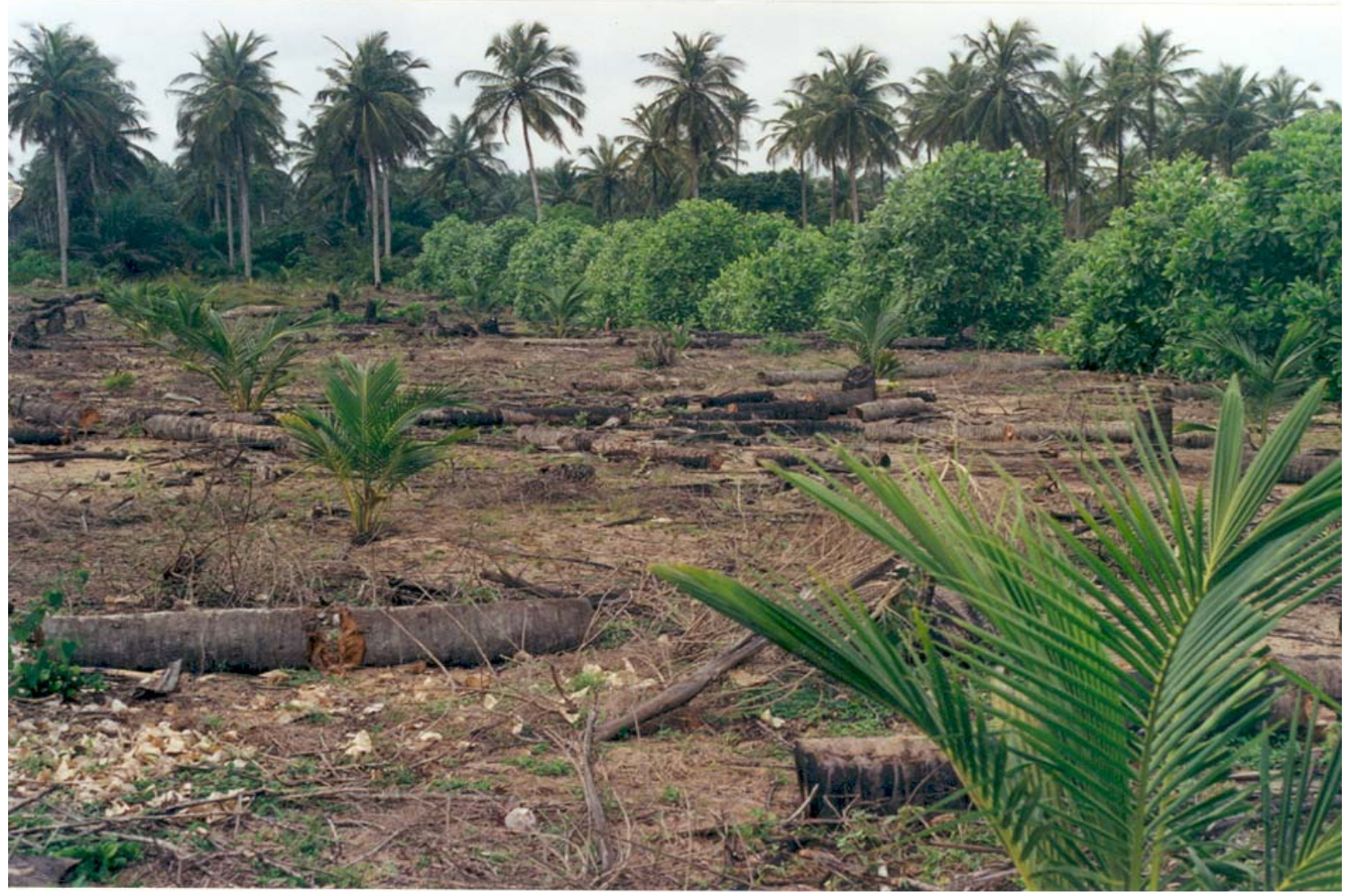

Figure 9 : Plantation d'Acacias australiens en mélange avec les cocotiers en zone littorale. (photo N'goran A.)

Australian acacias associated with coconut in the littoral zone. (photo N'goran A.) 


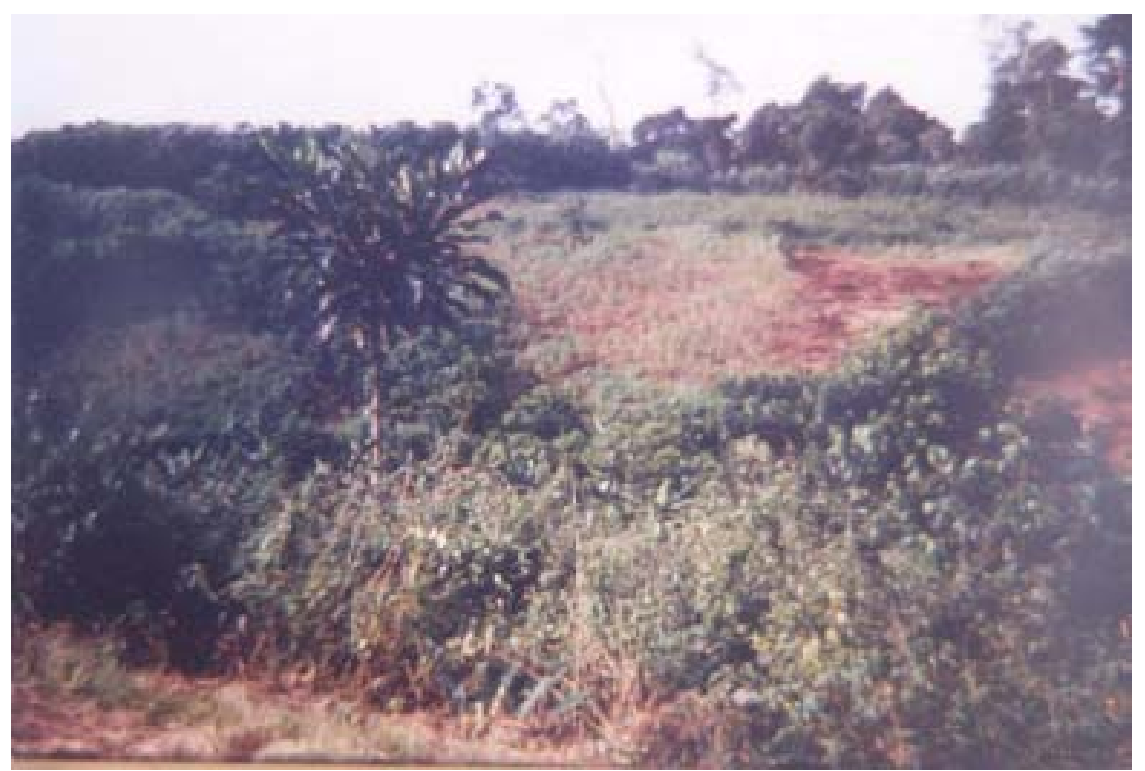

Figure 10 : Brise-vent d'Acacias australiens en zone forestière (Anguédédou).

Australian acacias as wind screen in forest zone (Anguédédou).

\section{DISCUSSION}

La sélection du matériel végétal a permis d'identifier 9 provenances performantes des Acacias australiens pour les reboisements sur les 25 testées. Ce sont : San Pédro, Oriomo River, Claudie River et Broken Pole Creck pour l'Acacia mangium, lokwa et Coën Cape pour l'Acacia auriculiformis, Wemenever et Oriomo River pour l'Acacia crassicarpa et la provenance d'Acacia aulacocarpa. Ces provenances identifiées dans les études de Cabaret et N'Guessan (1988) réalisées sur le littoral ont été confirmées par celles effectuées en zone de forêt dense humide sempervirente par Dupuy et N'Guessan (1989). Ces études ont montré aussi que les Acacias australiens sont aussi mieux adaptés sous les cocotiers en zone littorale du point de vue de la survie, de la croissance et de la résistance aux embruns de mer que les espèces des genres Albizia, Cassia et Leucaena. Les Albizias possèdent une bonne croissance certes, mais ils présentent une forte sensibilité aux embruns de mer.

Du point de vue de la croissance, on note une variabilité dans les comportements. Les croissances sont plus importantes en zone de forêt dense humide sempervirente que sur les sols sableux et pauvres du littoral. En général, l'Acacia mangium, l'Acacia crassicarpa et
l'Acacia aulacocarpa présentent de meilleures croissances (diamètre, hauteur) que l'Acacia auriculiformis quelle que soit la zone écologique. A partir de 3 années de plantation, l'apparition des effets de la concurrence entraîne une baisse de la croissance de toutes les espèces. De 4 à 7 ans, la production de bois est maximale et correspond à l'âge d'exploitabilité technique. Différer l'exploitation pendant cette période induit une perte de rendements en bois des peuplements malgré une augmentation du potentiel sur pied. L'influence de la densité de plantation sur le taux de tiges multiples qui représente un bon critère de production de bois de feu est sensible. II est de $43 \%$ pour la faible densité $\left(625\right.$ tiges. ha $\left.^{-1}\right)$ et de $7 \%$ pour la forte densité (1600 tiges.ha-1). La densité à la plantation de 1111 tiges.ha $^{-1}(3 \times 3 \mathrm{~m})$ a permis d'obtenir une production optimale de bois et des taux de tiges multiples raisonnés quelle que soit la zone écologique de plantation.

En dépit des attaques des Cérambycides dont Pakystola mamillata est le parasite principal, les arbres réagissent vigoureusement sans une mortalité sur pied ni une réduction de croissance significatives pour le moment. Mieux encore, des résultats préliminaires d'études menées dans de vieilles palmeraies signalent une baisse de la pression parasitaire de la fusariose sur les palmiers à huile imputable à la jachère d'Acacia mangium précédente (Allou, 2001). 
Les légumineuses arborescentes étudiées sont certes des espèces naturellement fixatrices d'azote, mais l'apport précoce d'une fertilisation stater au NPK (10-10-18) à la dose de 100 grammes/plant après la plantation stimule la croissance des arbres et permet d'obtenir rapidement un gain en volume de bois à deux ans de $23 \%$ chez l'Acacia mangium et $24 \%$ chez l'Acacia auriculiformis.

En reboisement, ces légumineuses arborescentes enrichissent les sols en matière organique par le truchement d'une forte production de litière. L'Acacia mangium produit une importante quantité de litière en comparaison avec les productions obtenues par Bernhard-Reversat (1976) dans les formations naturelles de Terminalia ivorensis (Framiré) de 22 ans en zone forestière de Côte d'Ivoire (9 t/ha.an $\left.{ }^{-1}\right)$, Lim (1988) dans des peuplements d'Acacia mangium de 4 ans en Malaisie (10 t/ha.an ${ }^{-1}$ ) et par Chijioke (1982) dans des reboisements de Gmelina arborea âgés de 5 à 6 ans au Brésil ( 1 à 4 t/ha.an ${ }^{-1}$ ). Des études réalisées par N'Guessan et Gnahoua (1998) en zone préforestière en Côte d'Ivoire dans un reboisement d'Acacia auriculiformis âgé de 8 ans donnent des résultats intéressants avec une production de litière de $6,5 \mathrm{t} / \mathrm{ha} \mathrm{an}^{-1}$. Les plus fortes chutes de biomasse aérienne sont observées en grande saison sèche (décembre à mars) et les plus faibles en grande saison des pluies (mai à juillet).

Concernant les traitements en taillis des légumineuses arborescentes, pour les périodes de recépage, les plus faibles mortalités de souches (10\%) sont enregistrées dans les peuplements d'Acacia mangium recépés en début de la grande saison des pluies (mars). Ces mortalités sont plus élevées (88 à $100 \%$ ) pour les recépages effectués en petite saison des pluies (septembre) et en grande saison sèche (décembre). En ce qui concerne les hauteurs de recépage, les mortalités les plus importantes (> $90 \%$ ) ont été observées au recépage rez-terre et les plus faibles (< $57 \%$ ) pour les recépages effectués à une hauteur supérieure à $0,50 \mathrm{~m}$ du sol. L'Acacia auriculiformis enregistre la plus faible valeur. Ces résultats sont encourageants en comparaison avec ceux obtenus ailleurs sur des espèces mondialement reconnues pour leur bonne capacité à rejeter telles que Eucalyptus sp. et Populus sp. (N'Guessan, 1990).

Associées aux cocotiers sur le cordon littoral ou plantées en brise-vents, les légumineuses arborescentes induisent une augmentation de la production en noix de coco, protègent les cultures contre les vents violents, l'ensoleillement excessif et l'érosion des sols et sécurisent l'occupation foncière. Comme corollaire, elles produisent de manière soutenue du bois de feu et de service. Mais lors de la phase d'installation des légumineuses arborescentes, la croissance en diamètre et en hauteur des plants est fortement ralentie par l'action des embruns de mer pendant les deux premières années de la plantation. Pendant cette période, les jeunes plants de l'Acacia mangium et de l'Acacia auriculiformis présentent des taux de brûlures des embruns de mer variant entre 20 et $60 \%$. Les plus faibles taux de brûlures sont enregistrés chez l'Acacia auriculiformis ( $\leq 30 \%$ ) et Casuarina equisetifolia ( $\leq 10 \%)$. Les arbres ont réagit en adoptant une forme buissonnante quantifiée par le rapport Diamètre houppier/Hauteur totale. Ce rapport varie entre 0,70 (2 ans) et 0,50 (3 ans) et baisse avec la bonne reprise de la croissance des arbres à trois ans après la période critique. L'utilisation des brise-vents et manchons protègent efficacement les plants contre les embruns sur une distance de 120 à $150 \mathrm{~m}$ pour l'Acacia mangium et 110 à $120 \mathrm{~m}$ pour Acacia auriculiformis. Les légumineuses arborescentes améliorent les rendements en noix de coco d'environ $50 \%$ par le biais d'un regain de fertilité des sols (Zakra, 1996).

Les plantations des légumineuses arborescentes comme brise-vents donnent une bonne production de bois chez toutes les espèces. On obtient une productivité de bois de $12 \mathrm{~m}^{3}$.ha-1/maille et à 8 ans, $30 \mathrm{~m}^{3}$.ha-1/maille. La croissance la plus faible est enregistrée chez l'Acacia auriculiformis. Au-delà de 3 ans, les brise-vents d'Acacias ont un effet dépressif sur les cultures de bordure. Cela occasionne une mortalité des cultures de bordure (effet de lisière) de maïs et de manioc. Cet effet est réduit puis annulé vers $10 \mathrm{~m}$ à l'intérieur des parcelles pour l'Acacia mangium et vers $6 \mathrm{~m}$ pour l'Acacia auriculiformis. 


\section{CONCLUSION}

Les études fournissent au secteur agricole en Basse Côte d'Ivoire, des méthodes de production plus simples, rentables et durables ainsi que de nouvelles sources de bois d'œuvre, d'énergie et de service, à courte révolution.

Les plantations de légumineuses arborescentes constituent des substituts efficaces de fertilisant de sols en Basse Côte d'Ivoire. Mais pour mieux valoriser cet intrant durable et peu agressif sur l'environnement, les études de la qualité de la litière doivent mettre l'accent sur la détermination des éléments minéraux effectivement libérés et utilisés pour chaque culture et améliorer la connaissance du processus de décomposition de la biomasse aérienne.

Avec des conditions particulières de recépage, les peuplements de légumineuses arborescentes étudiées peuvent être gérés en taillis pour le contrôle du développement rapide en hauteur des arbres et pour la production régulière et soutenue du bois de feu et service. La grande saison des pluies (mars à juin) est la période la plus indiquée pour ces opérations. Un dépressage des rejets épargnant deux ou trois brins est préconisé pour favoriser la production rapide de rejets vigoureux, permettre d'éviter aux gestionnaires, certains travaux de gestion (production de plants, désouchage, replantation) et pour maintenir un minimum de couvert forestier permanent sur les terrains exploités.

Les associations culturales avec le cocotier et en brise-vent avec les cultures annuelles (maïs, manioc, etc.) sont possibles. Elles permettent de régénérer les vieilles cocoteraies et de gérer de manière rationnelle et durable les exploitations agricoles et les propriétés foncières.

L'ampleur des dégâts causés par les Cérambycides dans les reboisements d'Acacias australiens n'est pas importante pour le moment. Mais il faut d'ores et déjà mettre l'accent dans les recherches sur une meilleure connaissance des parasites et les techniques de protection des reboisements.

Enfin, ces études contribuent de manière significative à la collecte des données sur les espèces agroforestières et forestières de substitution en prévision de l'établissement de leurs tables de production et leurs modèles de croissance importants pour la gestion durable de l'environnement des secteurs agricole et forestier en Côte d'Ivoire.

\section{REFERENCES}

Akomian (K.). 1988. Place des légumineuses enrichissants dans les jachères. Place de la jachère dans l'agriculture ivoirienne. Actes du $2^{\mathrm{e}}$ Atelier de l'OFRIC, ENSA : 22-28.

Allou (K.), Aké (S.), Ahoussou (N.), Ballo (K.) et (S.) Diabaté. 2001. Effet de la jachère sur l'expression de la Fusariose vasculaire du palmier à huile (Elaeis guineensis Jacq.). Agronomie Africaine, 13 (1) : 21 - 33.

Bernhard-Reversat (F.). 1976. Essai de comparaison des cycles d'éléments minéraux dans les plantations de Framiré (Terminalia ivorensis) en forêts naturelles de Côte d'Ivoire. Bois et Forêt des Tropiques, N'167 : 25 - 39.

Cabaret (N.) et (A.) N'Guessan. 1988. Essais comparatifs de provenances d'Acacia mangium et d'Acacia auriculiformis à Anguédédou et d'Acacia crassicarpa à l'IRHO (Port-Bouët). Rap. scien., CTFT Côte d'Ivoire, $8 \mathrm{p}$.

Chijioke (E. O.). 1982. Influences exercées par les essences à croissance rapide sur les sols des régions tropicales humides de plaine. Doc. FAO, 21, 109 p.

Dommergues (Y.) ; Duhoux (E.) et (D. G.) Hoang. 1999. Les arbres fixateurs d'azote (Caractéristiques fondamentales et rôles dans l'aménagement des écosystèmes méditerranéens et tropicaux). Edition CIRAD, FAO, IRD, 499 p.

Dupuy (B.) et (A.) N'Guessan. 1989. Essais comparatifs de provénances d'Acacias australiens : Acacia mangium, Acacia auriculiformis, Acacia crassicarpa en Basse Côte d'Ivoire. Rap. scien., CTFT, 10 p.

Dupuy (B.) et (A.) N'guessan. 1990. Sylviculture de l'Acacia mangium en Basse Côte d'Ivoire. Bois et Forêts des Tropiques, $\mathrm{N}^{\circ} 225,3^{\mathrm{e}}$ trimestre : $24-32$.

Dupuy (B.) et (A.) N'Guessan. 1991. Utilisation des Acacias pour régénérer les anciennes cocoteraies. Bois et Forêts des Tropiques, $\mathrm{N}^{\circ} 230,4^{\mathrm{e}}$ trimestre, p. $15-29$.

Lim (M. T.). 1988. Studies of Acacia mangium in Kemasul Forest Malasia. I. Biomass and productivity. Journal of tropica Ecology, IUFRO, 4 (3) : 293 - 302.

Mallet (B.). 1988. Pakystola mamillata, Cérambycide déprédateur d'Acacia mangium en Côte d'Ivoire. Rap. scien., CTFT Côte d'Ivoire, 5 p. 
National Academy of Sciences. 1980. Firewood crops. Shrub and Tree Species For Energy Production, Volume 1, Washington, D.C., $236 \mathrm{p}$.

N'Diaye (S.). 1991. Croissance et productivité d'Acacia auriculiformis en Côte d'Ivoire. Mém. de fin d'Etude d'Ingénieur agronome, IDEFOR-DFO/ENSA, Côte d'Ivoire,75 p

N'Goran (A.). 2005. Amélioration de la fertilité chimique des sables quaternaires en Côte d'Ivoire dans l'association cocotier/Acacias $s p$. Thèse de Doctorat, Univ. de GAND, Belgique, $193 \mathrm{p}$.

N'Guessan (A.). 1990. Sylviculture en taillis à courtes révolutions, carbonisation et mycorhization. Rap. de stage de formation, CTFT Nogent/AFOCEL/CTFT Côte d'Ivoire, $59 \mathrm{p}$.

N'Guessan (A.). 1991. Contribution à l'étude des méthodes de régénération des jachères en Basse Côte d'Ivoire : le cas de l'Acacia mangium. Thèse de Doctorat-Ingénieur, Univ. Nationale de Côte d'Ivoire, 128 p.

N'Guessan (A.). 1996. Les brise-vents d'Acacias australiens, un système intégré d'aménagement des terrains agricoles. Rap. scien., IDEFOR-DFO Côte d'Ivoire, 6 p.
N'Guessan (A.) et (G. M.) Gnahoua. 1998. Production de matière organique par les légumineuses arborescentes en zone forestière de Côte d'Ivoire. Association Africaine de la Fixation de l'Azote. In : DAKORA (F.) (D.), Edition UE/FAO/CTA/ CSC, Actes de l'Atelier sur la fixation biologique de l'azote en Afriquede processus au progrès, Cap, Afrique du Sud : 179 - 183.

Offi (K). 1988 Bilan en 1988 des essais mis en place dans le Sud-Ouest (San-Pédro) de la Côte d'Ivoire. Rap. scien., CTFT, Côte d'Ivoire, $30 \mathrm{p}$.

Oliuver (R.) et (F.) Ganry. 1994. Etude des modifications de fertilité induites par une jachère arborée. Cas de la zone forestière du Centre Côte d'Ivoire. Compte rendu de fin d'études d'une recherche financée par le Ministère de la Recherche et de la Technologie, CIRAD, $27 \mathrm{p}$.

Stewart (J. L.), Dundon (A. J.), Hellin (J. J.) et (C. E.) Hugues. 1992. Wood Biomass Estimation of Central American dry zone hardwood species.OFI Oxford, $83 p$

Zakra (N.). 1996. Bilan positif de l'association Cocotier/Acacias pour la restitution de l'Azote. Plantation Recherche Développement, $18 \mathrm{p}$. 